\title{
SPIN NETWORKS AND THE BRACKET POLYNOMIAL
}

\author{
LOUIS H. KAUFFMAN \\ Department of Mathematics, Statistics and Computer Science \\ University of Illinois at Chicago \\ 851 South Morgan Street, Chicago, Illinois 60607-7045, U.S.A. \\ E-mail: kauffman@uic.edu
}

\begin{abstract}
This paper discusses Penrose spin networks in relation to the bracket polynomial.
1. Introduction. This paper is an introduction to the relationship between Penrose spin networks and the bracket polynomial. The paper is organised as follows. Section 2 recalls the bracket model for the Jones polynomial invariant of knots and links. In Section 3 we show how the bracket state model is a natural generalization of the original Penrose spin networks, and how this model is related to the quantum group corresponding to $S L(2, C)$. In particular, we show how the binor identity of the spin networks, the skein identity of the bracket polynomial (at a special value) and the trace identity

$$
\operatorname{tr}(A B)+\operatorname{tr}\left(A B^{-1}\right)=\operatorname{tr}(A) \operatorname{tr}(B)
$$

of $S L(2, C)$ are really all the same. The section continues with a discussion of the role of these generalized networks in low dimensional topology. In particular we discuss the relationship of these nets with the evaluation of the Witten-Reshetikhin-Turaev invariant of 3 -manifolds and corresponding relations with quantum gravity theories (Regge-Ponzano in $2+1$ and Ashtekar-Smolin-Rovelli in $3+1$ ).
\end{abstract}

Acknowledgements. It gives the author pleasure to thank the National Science Foundation for support of this research under NSF Grant DMS-2528707.

2. Recalling the bracket state summation and the Jones polynomial. The bracket state summation [10], [11], is a model for the original Jones polynomial [9] as a partition function defined in terms of combinatorial states of the link diagram.

This partition function will be referred to as the bracket state model for the Jones polynomial. It is the purpose of this section to describe the bracket model. The next section will show how this model is a perspicuous generalisation of the binor calculus of

1991 Mathematics Subject Classification: Primary 57M.

The paper is in final form and no version of it will be published elsewhere. 
Roger Penrose [17]. The binor calculus forms the underpinning of the Penrose theory of spin networks [18], and is directly related to the group $S L(2, C)$.

The bracket polynomial is based on the structure of the two smoothings at a crossing in a knot diagram. At a given crossing there are four local regions. Call two out of the four local regions a pair if they meet only at the vertex. Call the pair that are swept out by a counterclockwise turn of the overcrossing a line the $A$-pair. Call the remaining pair the B-pair. The A-smoothing is the smoothing that joins the local regions of the A-pair. The $B$-smoothing is the smoothing that joins the local regions of the B-pair. See Figure 1 for an illustration of this basic distinction.

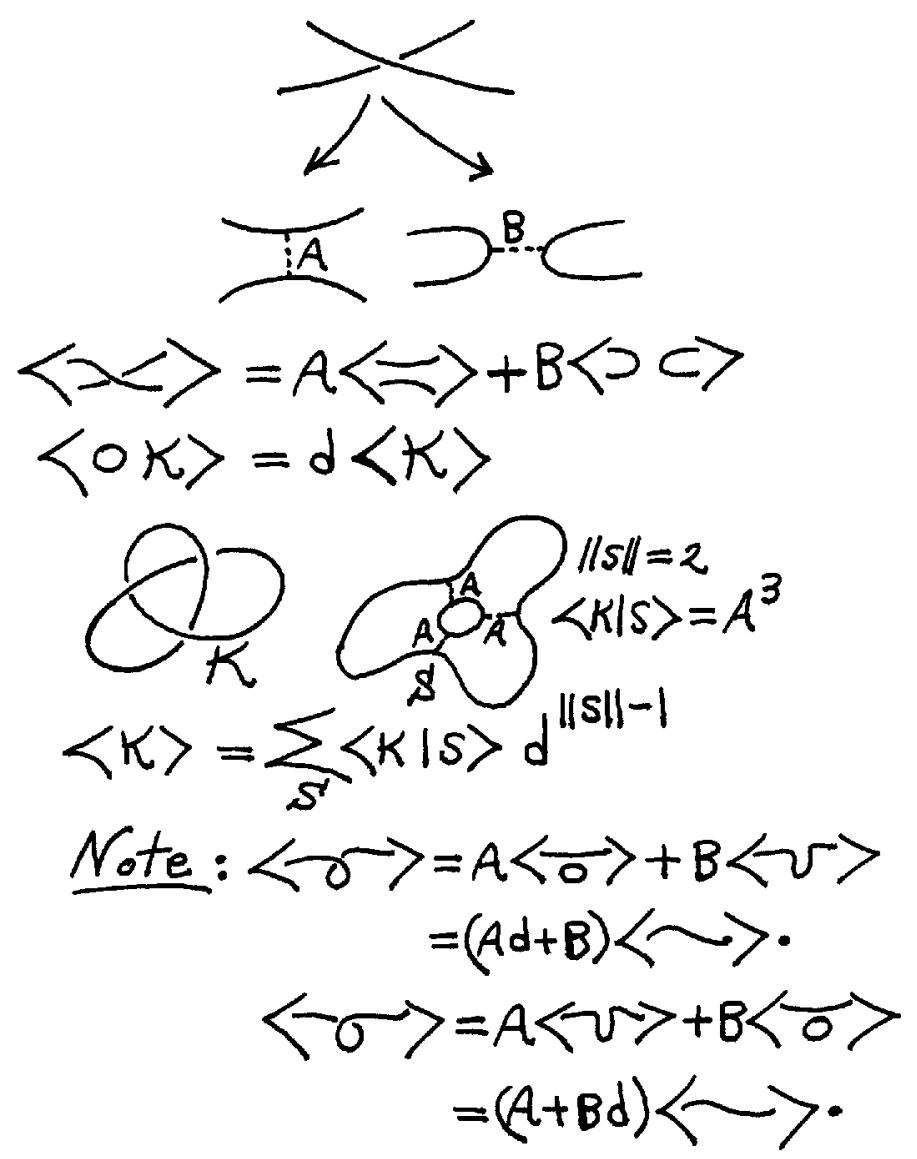

Fig. 1. Smoothings and bracket expansion

The three-variable bracket polynomial is defined on link diagrams by the following formulas:

1. $\langle K\rangle=A\left\langle K_{1}\right\rangle+B\left\langle K_{2}\right\rangle$ where $K_{1}$ and $K_{2}$ are two diagrams obtained from a given crossing in $K$ by smoothing the crossing in the two possible ways illustrated in Figure 1. with an $A$-smoothing in $K_{1}$ and a $B$-smoothing in $K_{2}$. 
2. $\langle K \bigsqcup D\rangle=d\langle K\rangle$ where $D$ denotes any Jordan curve that is placed into the complement of the diagram $K$ in the plane.

3. $\langle D\rangle=1$ for any Jordan curve $D$ in the plane.

Here the small diagrams stand for otherwise identical parts of larger diagrams, and the second formula means that any Jordan curve disjoint from the rest of the diagram contributes a factor of $d$ to the polynomial. This recursive description of the bracket is well-defined so long as the variables $A, B$ and $d$ commute with one another.

The bracket can be expressed as a state summation where the states are obtained by smoothing the link diagram in one of two ways at each crossing. A smoothing of type $A$ contributes a vertex weight of $A$ to the state sum. A smoothing of type $B$ contributes a vertex weight of $B$ to the state sum. The norm of a state $S$, denoted $\|S\|$, is defined to be the number of Jordan curves in $S$. It then follows that the bracket is given by the formula

$$
\langle K\rangle=\Sigma_{S}\langle K \mid S\rangle d^{|| S||-1} .
$$

where the summation is over all states $S$ of the diagram $K$, and $\langle K \mid S\rangle$ denotes the product of the vertex weights for the state $S$ of $K$.

In the variables $A, B$ and $d$ the bracket polynomial is not invariant under the Reidemeister moves. However the following Lemma provides the clue to finding a specialization of the polynomial that is an invariant of regular isotopy, the equivalence relation generated by the second and third Reidemeister moves [20].

Lemma. Let $K_{* *}$ denote a diagram with the local configuration shown in Figure 2. Let $K_{v}$ and $K_{h}$ denote the two local smoothings of this configuration as shown also in Figure 2. Then

$$
\left\langle K_{* *}\right\rangle=A B\left\langle K_{v}\right\rangle+\left(A B d+A^{2}+B^{2}\right)\left\langle K_{h}\right\rangle .
$$

Proof. The proof is illustrated in Figure 2.

Since $K_{v}$ is obtained from $K_{* *}$ by a second Reidemeister move, it follows that $\langle K\rangle$ can be made invariant under the second Reidemeister move if we take $B=A^{-1}$, and $d=-A^{2}-A^{-2}$. In fact, with this specialization, $\langle K\rangle$ is also invariant under the third Reidemeister move, and it behaves multiplicatively under the first Reidemeister move. This allows the normalization

$$
f_{K}=\left(-A^{3}\right)^{-w(K)}\langle K\rangle
$$

$f_{K}$ is an invariant of ambient isotopy for links in three space. Here $w(K)$ is the sum of the signs of the crossings of the oriented link $K$.

TheOREM [10]. $f_{K}\left(t^{-1 / 4}\right)=V_{K}(t)$ where $V_{K}(t)$ is the original Jones polynomial [9].

3. Spin networks. The original Penrose spin networks [17], [18] were devised to create a diagrammatic and combinatorial substrate for the recoupling theory of quantum mechanical angular momentum. The key to this diagrammatics is a system of abstract tensors based on the properties of a classical epsilon (the definition will be given below) and adjusted to obtain topological invariance under planar deformations of the diagrams. 
It is this adjustment in the direction of topological invariance that makes these networks a special case of the bracket state model for the Jones polynomial [12].

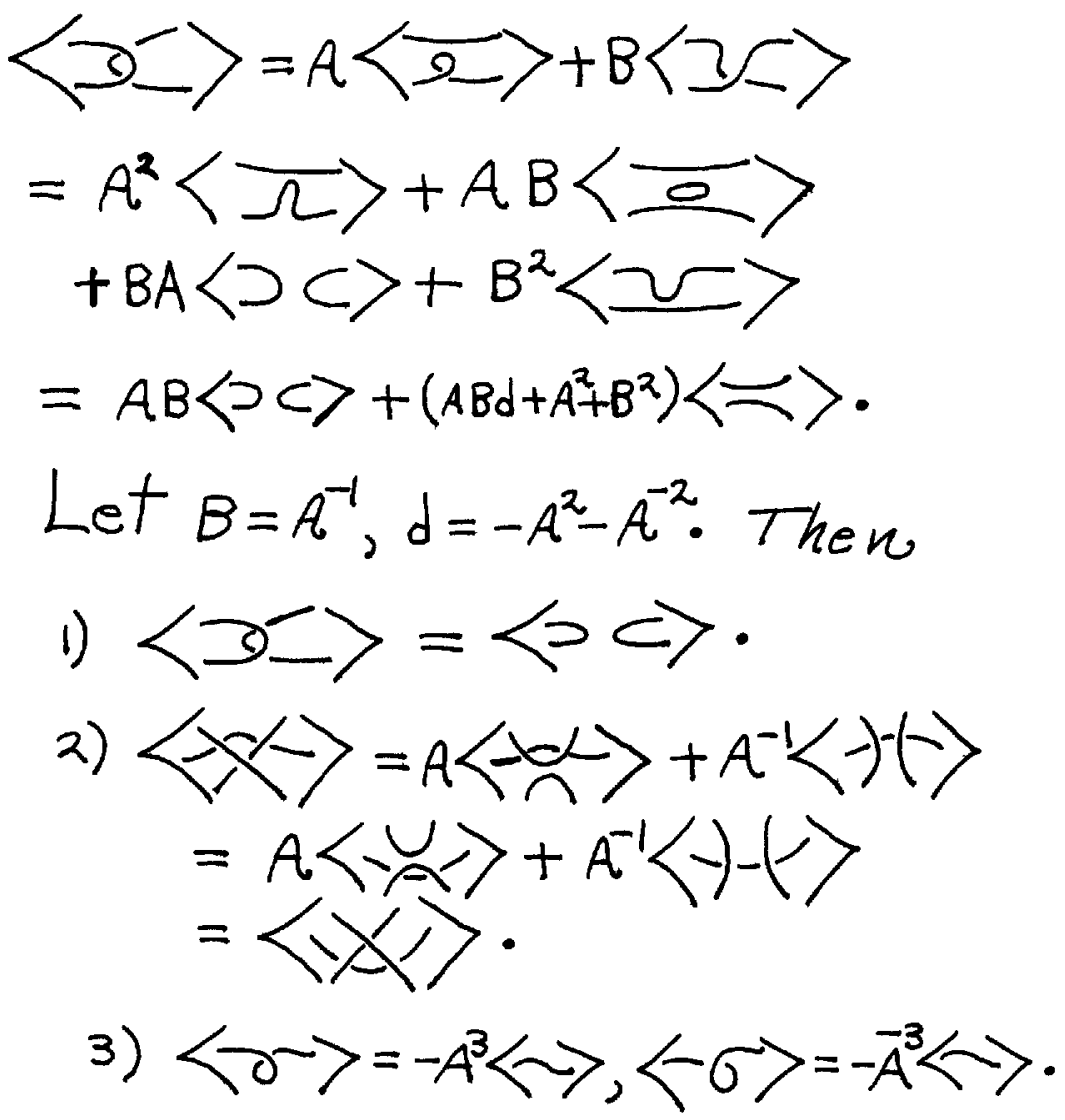

Fig. 2. Bracket invariance

The classical epsilon is defined by the equations $\epsilon_{12}=1, \epsilon_{21}=-1$ and $\epsilon_{i j}=0$ if $i=j$. The indices are in the set $\{1,2\}$. Note that the Kronecker delta is defined by the equation $\delta_{a b}=\delta^{a b}=\delta_{a}^{b}=1$ if $a=b$ and 0 if $a \neq b$. Epsilons and deltas are related by the fundamental equation

$$
\epsilon^{a b} \epsilon_{c d}=\delta_{c}^{a} \delta_{d}^{b}-\delta_{d}^{a} \delta_{c}^{b}
$$

We shall refer to this equation as the epsilon identity. It is natural to diagram this relation by letting a vertical line represent a single Kronecker delta, a cup (a local minimum, see Figure 3) represent an epsilon with upper indices, a cap (a local maximum, see Figure 3) represent an epsilon with lower indices. Note that in such a convention $\delta_{c}^{a} \delta_{d}^{b}$ is represented by vertical parallel lines, while $\delta_{d}^{a} \delta_{c}^{b}$ is represented by vertical crossed lines. The key to topological invariance is that the cups and the caps should satisfy the cancellation to a delta as in

$$
C A P_{a i} C U P^{i b}=\delta_{a}^{b}
$$


(with implicit summation over $i$ ). This is accomplished in this formalism with

$$
C A P_{a b}=i \epsilon_{a b}, \quad C U P^{a b}=i \epsilon^{a b} .
$$

Here $i^{2}=-1$. Finally it is convenient to introduce a minus sign at the the crossing of two diagrammatic lines. With these conventions we obtain the binor identity as illustrated in Figure 3.

$$
\begin{aligned}
& T_{a} T_{b}=\varepsilon_{a b}, \quad \underline{1}^{b}=\varepsilon^{a b} \\
& \varepsilon^{a b} \varepsilon_{c d}=\delta_{c}^{a} \delta_{d}^{b}-\delta_{d}^{a} \delta_{c}^{b} \\
& \left.\frac{\| 1}{\pi}=\right)(-X \\
& \hat{U}^{a}=i \varepsilon^{a b}=i \Perp^{b} \\
& \bigcap_{a}=i \varepsilon_{a b}=i \prod_{b} \\
& \chi_{c}^{a}{ }_{d}^{b}=-{ }_{c}^{a} X_{d}^{b}=-\delta_{d}^{a} \delta_{c}^{b} \\
& \left.\Rightarrow U_{n}=i^{2} \frac{\|}{\pi}=-\right)(+X \\
& \left.\Rightarrow \bigcup_{\cap}+\right)(+X=\phi \text { binor } \\
& \Theta_{b}=\sum_{a, b}\left(a b=\sum_{a, b} \dot{i}^{2} \varepsilon_{c b} \varepsilon^{a b}=-2\right. \text {. }
\end{aligned}
$$

Fig. 3. Epsilons and the binor identity

Notational warning. We have used $i$ both as a matrix index and as the notation for the square root of minus one. This difference is clear from context - the square root of minus one never occurs as an index, the index $i$ never occurs as a separate variable!

The reader will note that the binor identity is exactly the exchange identity for the bracket polynomial when $A=-1=B$ and $d=-A^{2}-A^{-2}=-2$. Note particularly that the loop value -2 coincides with the loop value in abstract tensors as shown in Figure 3 . This loop value is $\Sigma_{a b} C U P^{a b} C A P_{a b}=-1-1=-2$ The binor identity is the basis of the original Penrose spin nets. 
The use of the epsilon tensor in the spin networks is directly related to the group $S L(2, C)$. The algebraic reason for this is that for any $2 \times 2$ matrix $P$ with commuting entries,

$$
P \epsilon P^{t}=\operatorname{det}(P) \epsilon
$$

where $\epsilon$ is regarded as a $2 \times 2$ matrix, and $P^{t}$ denotes the transpose of the matrix $P$. Thus $S L(2, C)$ is the set of $2 \times 2$ matrices $P$ over $C$ such that

$$
P \in P^{t}=\epsilon \text {. }
$$

The $\epsilon$ identity that is at the basis of the binors is then easily seen to be the source of the following fundamental lemma about $S L(2, C)$ :

LEMmA. If $A$ and $B$ are matrices in $S L(2, C)$ and tr denotes the standard matrix trace, then

$$
\operatorname{tr}(A B)+\operatorname{tr}\left(A B^{-1}\right)=\operatorname{tr}(A) \operatorname{tr}(B)
$$

Proof. In this proof we will use the Einstein summation convention for repeated indices (i.e. we sum over all values in the index set $\{1,2\}$ whenever an index occurs twice in a given expression). Note that if $A$ is in $S L(2, C)$, then

$$
A \epsilon A^{t}=\epsilon \text {. }
$$

Note that

$$
\epsilon^{-1}=-\epsilon
$$

Therefore

$$
A=-\epsilon\left(A^{t}\right)^{-1} \epsilon
$$

Hence

$$
\begin{aligned}
\operatorname{tr}(A B) & =A_{i k} B_{k i}=-A_{i r} \epsilon_{r s}\left(\left(B^{t}\right)^{-1}\right)_{s l} \epsilon_{l i}=-A_{i r}\left(\left(B^{t}\right)^{-1}\right)_{s l}\left(\epsilon_{r s} \epsilon_{l i}\right) \\
& =-A_{i r}\left(\left(B^{t}\right)^{-1}\right)_{s l}\left(\delta_{r l} \delta_{s i}-\delta_{r i} \delta_{s l}\right) \\
& =-A_{i r}\left(\left(B^{t}\right)^{-1}\right)_{s l} \delta_{r l} \delta_{s i}+A_{i r}\left(\left(B^{t}\right)^{-1}\right)_{s l} \delta_{r i} \delta_{s l} \\
& =-A_{i l}\left(\left(B^{t}\right)^{-1}\right)_{i l}+A_{i i}\left(\left(B^{t}\right)^{-1}\right)_{l l}=-\operatorname{tr}\left(A B^{-1}\right)+\operatorname{tr}(A) \operatorname{tr}\left(B^{-1}\right) .
\end{aligned}
$$

This completes the proof.

R e m a r k. This lemma shows that the $\mathrm{SL}(2)$ identity $\operatorname{tr}(A B)+\operatorname{tr}\left(A B^{-1}\right)=\operatorname{tr}(A) \operatorname{tr}(B)$ is essentially a matrix algebraic expression of the binor identity, and hence equivalent to the corresponding identity for the bracket polynomial at the special value $A=-1$. This explains the relationship of the bracket polynomial with $S L(2, C)$ that is found in the work of Bullock [1]. It also suggests the possibility of generalising Bullock's work to the corresponding quantum group, just as the introduction to the variable $A$ into the bracket polynomial corresponds to the movement from $S L(2, C)$ to the quantum group (See [13] for more details on the relationship of the bracket polynomial to the $S L(2)$ quantum group.)

In Figure 4 we illustrate how a deformation of the epsilon matrix, via the variable $A$, leads to a model of the bracket polynomial. It is customary to call this a $q$-deformation where $\sqrt{ } q=A$. In the deformation, $\epsilon$ is replaced by $\tilde{\epsilon}$, a matrix that is a function of $A$. 
The quantum group $S L(2)_{q}$ arises by generalising the equation $P \epsilon P^{t}=\epsilon$ to

$$
P \tilde{\epsilon} P^{t}=\tilde{\epsilon} \quad \text { and } \quad P^{t} \tilde{\epsilon} P=\tilde{\epsilon} .
$$

The entries of $P$ become elements of a non-commutative algebra. It is this algebra that is called $S L(2)_{q}$. As illustrated in Figure 4, the binor identity generalises to the smoothing identity for the bracket polynomial, and the crossing of lines deforms to the under or over crossing of strands in a knot diagram.

$$
\begin{aligned}
& M=\left[\begin{array}{cc}
\phi & i A \\
-i A^{-1} & \phi
\end{array}\right]=\dot{\widetilde{\varepsilon}}, \sqrt{q}=A \\
& M^{a b}=M_{a b}, \varepsilon=\widetilde{\varepsilon}(A=-1) \\
& u^{a}=M^{a b}, \bigcap_{b}=M_{a b} \\
& \mu^{2}=\left.\left.I \Rightarrow\right|_{a} ^{i}\right|^{b}=\sum_{i} M_{a i} \mu^{i b}=\delta_{a}^{b}=\left.\right|_{a} ^{b} \\
& \text { @b }=\sum_{a, b} M_{a b} M^{a b}=\sum_{a, b}\left(M_{a b}\right)^{2}=-A^{2}-A^{-2} \text {. } \\
& \left.\lambda_{d d}^{b}=A \bigcup_{a}^{a} b+A^{-1}\right)_{c}^{a}(b \\
& \left.\sum_{c}^{a} /_{d}^{b}=A^{-1} \hat{\bigcap}_{c}^{b}+A\right)_{d}^{a}\left(\begin{array}{l}
b \\
d
\end{array}\right\} \\
& q \text {-deformed binor identities }
\end{aligned}
$$

Fig. 4. Bracket polynomial and deformed epsilons

3.1. Classical spin networks. This subsection is a quick resume of the classical theory of (Penrose) spin networks. As we have seen, the binor identity (Figure 3) can be regarded as an expression of the basic epsilon identity

$$
\epsilon^{a b} \epsilon_{c d}=\delta_{c}^{a} \delta_{d}^{b}-\delta_{d}^{a} \delta_{c}^{b}
$$

In the binor expression for the epsilon identity the diagrams can be manipulated topologically in the plane, without changing the values of the corresponding evaluations. The topological manipulation that we refer to is regular homotopy augmented by a projection 
of the first Reidemeister move. This provides the basis for a more complex theory of network evaluations.

A key ingredient in the full theory is the use of network lines that stand for multiplicities of parallel strands. Such a line is labelled with a positive integer to indicate the multiplicity of strands. Then permutations, symmetrizers and antisymmetrizers are introduced. A permutation takes the form of the projection of a braid to the plane. That is, a permutation (as a network) consists in $n$ start points and $n$ end points. Each strand in the network connects one start point to a unique endpoint. The mapping of start points to end points is the corresponding permutation. The strands can cross one another in the planar representation, and being subject to augmented regular homotopy, really only represent this permutation. Since the binor identity allows us to resolve a crossing in a permutation, we see that the permutation network can be rewritten as a sum of flat tangles that have no crossings. See Figure 6 . The permutation is expressed in terms of the diagrammatic Temperley Lieb algebra [13]. In the original Penrose nets, the expansion into networks free of crossings was central to the network evaluations.

$$
\begin{aligned}
& S_{2}: \|, X \\
& S_{3}: I I, X|,| X, X, X, \mathbb{X} \\
& l_{b}^{a}=\delta_{b}^{a}, \operatorname{tr}(1)=\sum \delta_{a}^{a}=N \\
& \frac{111}{B]} \Rightarrow \operatorname{tr}(B)=\sqrt{B]}=\sum_{a, b, c} B_{a b c}^{a b c} . \\
& \operatorname{tr}(\| I)=\square=N^{3} \\
& \operatorname{tr}(x)=50 N \\
& {[[n]]=\prod_{1}^{n}=\sum_{\sigma \in S_{n}} \frac{1}{n !}(-1)^{t(\sigma)} \frac{1}{\sigma}} \\
& \operatorname{tr}[[n]]=C_{n}^{N}=\frac{N(N-1) \cdots(N-n+1)}{n !} \\
& \text { e.g. } \left.\operatorname{tr}[[2]]=\frac{1}{2 !}(\square]-\square\right) \\
& =\frac{1}{2 !}\left(N^{2}-N\right)=\frac{N(N-1)}{2 !}=C_{2}^{N} \text {. }
\end{aligned}
$$

Fig. 5. Permutations and traces 


$$
\begin{aligned}
& N=-2=\operatorname{Value}(0) \text {. } \\
& \left.\begin{array}{l}
T^{n}=\frac{1}{n !} \sum_{\sigma \in S_{n}}(-1)^{t(\sigma)} \frac{1}{\sigma^{\prime}} \\
\left.X=-\bigcup_{n}^{-}-\right)(.
\end{array}\right\} \\
& \left\{\begin{aligned}
\text { e.g.g. } \frac{1}{1}^{2} & =\frac{1}{2}(1(-X) \\
& =\frac{1}{2}()\left(+U_{n}+\right)() \\
\|_{11}^{\prime \prime} & =)\left(+\frac{1}{2} U_{n}\right.
\end{aligned}\right\}
\end{aligned}
$$

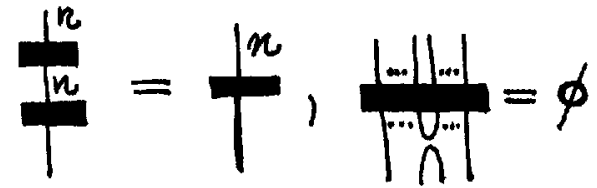

$$
\begin{aligned}
& \text { e.g. } \frac{\|}{n}=U_{n}^{U}+\frac{1}{2}{\underset{O}{U}}_{n}^{U}=\frac{U}{2}+\frac{1}{2}(-2)^{U} \\
& =\phi \text {. }
\end{aligned}
$$

Fig. 6. Antisymmetrizers

A symmetrizer or antisymmetrizer is a formal sum of permutations with appropriate coefficients. Thus if $[\sigma]$ denotes the network permutation corresponding to the permutation $\sigma$ in the symmetric group $S_{n}$, then we denote by $[[n]]$ the antisymmetrizer net

$$
[[n]]=\Sigma_{\sigma \epsilon S_{n}}(-1)^{t(\sigma)}[\sigma]
$$

where $t(\sigma)$ denotes the least number of transpositions needed to transform $\sigma$ to the identity permutation. See Figures 5 and 6 . In Figure 5 we illustrate properties of these nets where the only tensors under consideration are Kronecker deltas. In this formalism the trace of a matrix is diagrammed by connecting the top and the bottom of the diagrammatic tensor. Figure 5 shows how, with an integer $N$ valued loop (positive $N$-dimensional underlying vector space) the trace of the antisymmetrizer is given by the formula

$$
\operatorname{tr}([[n]])=C_{n}^{N}
$$

where $C_{n}^{N}$ denotes the binomial coefficient that counts the number of choices of $n$ items from $N$ items. Letting $N=-2$, we obtain the trace value for the corresponding antsym- 
metrizer in the binor calculus. The result is:

$$
\begin{aligned}
C_{n}^{N} & =N(N-1)(N-2) \ldots(N-n+1) / n ! \\
C_{n}^{-2} & =(-2)((-2)-1)((-2)-2) \ldots((-2)-n+1) / n ! .
\end{aligned}
$$

Hence

$$
C_{n}^{-2}=(-2)(-3)(-4) \ldots(-(n+1)) / n !=(-1)^{n}(n+1) .
$$

At the loop value -2 the trace of the antsymmetrizer on $n$ lines is the integer $(-1)^{n}(n+1)$. The reader may glimpse from this example why Penrose called the binors a calculus of "negative-dimensional" tensors! The trace calculation that we have shown is a miniature example of the so called chromatic method of spin network evaluation. In making the transition to the $q$-deformed networks, the integer value of $C_{n}^{-2}$ is replaced by a "quantum integer". See [14] for the details of this transition.

$$
\begin{aligned}
& \left\{_{c}^{a}=\sum_{\left.\right|_{c} ^{j}}^{a}\right. \\
& i+j=a) \quad i=\frac{1}{2}(a+b-c) \\
& i+k=b \quad \Rightarrow j=\frac{1}{2}(a+c-b) \\
& j+k=c\} \begin{array}{l}
j=\frac{1}{2}(a+c-b) \\
k=\frac{1}{2}(b+c-a)
\end{array} \\
& \begin{aligned}
0 & =\operatorname{tr}\left([[x])=C_{n}^{-2}\right. \\
& =(-2)(-2-1) \cdots(-2-x+1) / x ! \\
& =(-2)(-3) \cdots(-n-1) / x ! \\
& =(-1)^{x+1}(n+1) \\
n & =\Delta_{n},(A=-1) .
\end{aligned}
\end{aligned}
$$

Fig. 7. Three-vertices

With the machinery of the permutation nets, we can define three-vertices. A threevertex is a meeting of three multiple lines with multiplicities $a, b$ and $c$. These lines are 
interconnected according to the schema shown in Figure 7. Each line contains an antisymmetrizer, and the lines emanating from the three antisymmetrizers are interconnected as shown in Figure 7. This entails the condition that $a+b+c$ is even and that the sum of any two members of the set $\{a, b, c\}$ is greater than the value of the remaining third member. A spin network is a trivalent graph (possibly with some free ends) such that each vertex is assigned a specific cyclic order, and each edge is labelled with a natural number. (An edge labelled with zero can be deleted from the net.)

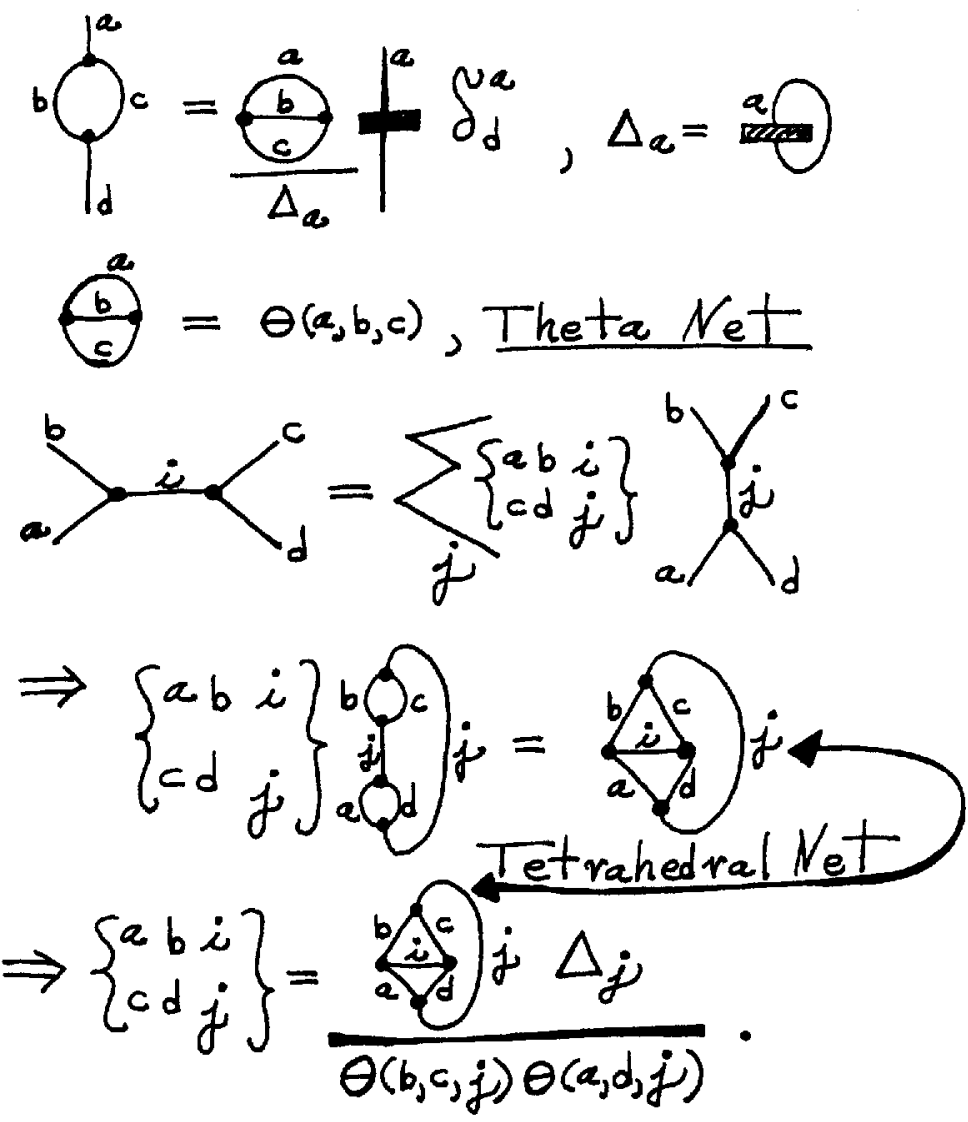

Fig. 8. Special nets and recoupling

Spin networks without free ends are evaluated by expanding them as sums of signed products of loop evaluations. Each loop evaluates to -2 . Such direct evaluations can be done in principle, but in practice it is useful to have a calculus of network recombination to help in the evaluations. In Figure 8 I have indicated some of the main features of this recombination calculus. These formulas insure that any network can be evaluated by just knowing the values of the "theta" and "tetrahedral" nets (as illustrated in Figure 8). The recombination formulas are a direct analogue of corresponding structures in the theory of angular momentum associated with the groups $S U(2)$ and $S L(2, C)$. In fact, the three- 
vertex, as we have described it, is a combinatorial version of taking the tensor product of two irreducible representations of $S U(2)$, and projecting the result into an irreducible factor of the tensor product. The spin network calculus shows that this theory, based on $S U(2)$ has a completely combinatorial expression that is intimately related to the topology of plane nets (via the binor identity).

One of the most interesting features of the classical spin nets is that they can be evaluated by a method that counts certain colorings of the loops in the graph of the network. These "chromatic evaluations" of the spin nets were originally described by Penrose and Moussouris in [16]. The reader can find an accounting of this point of view in [14] along with a comparison of the analogous situation for the $q$-deformed spin networks that we use in studying invariants of three-manifolds. It is not obvious how to fully generalise the chromatic method to the $q$-deformed spin nets.

4. Spin geometry and the geometry of spin networks. The bracket polynomial provides a natural generalization or deformation of the classical spin networks where the polynomial variable A becomes a deformation parameter. In fact, this mode of generalization carries over on all levels of the structure. The group $S L(2, C)$, naturally associated with the spin networks is generalized to a corresponding quantum group (Hopf algebra). The flat networks projected into the plane become woven networks in three dimensional space. The symmetrizers and apparatus of recoupling theory have braided analogs that can be expressed purely diagrammatically and in terms of the quantum groups.

In Figure 9 we have indicated some of the changes that occur in deforming the classical spin nets. In particular, we have illustrated the definition of the deformation of the antisymmetrizer as a sum over permutations that are lifted to braids. For the details of this theory the reader can consult [13], [14], [2], [5], [3].

One of the key facts about the classical spin networks is the Penrose Spin Geometry Theorem [17], [16]. This theorem states that for sufficiently large and well-behaved networks, the properties of three dimensional space (in particular, properties of angles) emerge of their own accord from the network structure. In this way the networks provide a combinatorial background for the emergence of properties of space and time. The emergence of space-time from such networks is not yet fully articulated.

More precisely, the Spin Geometry Theorem gives a method of determining an "angle" between two subnetworks of the larger network by considering the results of exchanges of spin between the subnetworks in relation to global network evaluations. The theorem states that these angles obey the dependency relations expected of angles in three dimensional Euclidean space. It is in this way that the angular properties of three dimensional space begin to emerge from the combinatorics of the nets.

With the Spin Geometry Theorem in mind, it is quite significant to see what is added by going to the topological spin nets with their deformation parameter $A$. When $A$ is a root of unity, the topological networks can be used to describe invariants of three dimensional manifolds. These invariants are defined in terms of surgery on links in three space, but the prescription of the three manifold structure is actually purely combinatorial in terms of the link diagrams. The evaluation of these diagrams in terms of topological spin 
networks is accomplished via generalised recoupling theory, and it expresses everything in terms of evaluations of trivalent networks with appropriate spin assignments on their edges. In this way, certain small spin networks encode deep topological properties of three dimensional manifolds. These results should be regarded as adding another dimension to the philosophy behind the Spin Geometry Theorem.

$$
\begin{aligned}
& \left.X=A_{n}^{U}+A^{-1}\right)(=X \\
& \left.\right|^{n}=\frac{1}{\{x\} \mid} \sum_{\sigma \in S_{n}}\left(A^{-3}\right)^{t(\sigma)} \frac{1}{\sigma} \\
& \{n\} \mid=\sum_{\sigma \in S_{n}}\left(A^{-4}\right)^{t\left(\sigma^{-}\right)}
\end{aligned}
$$

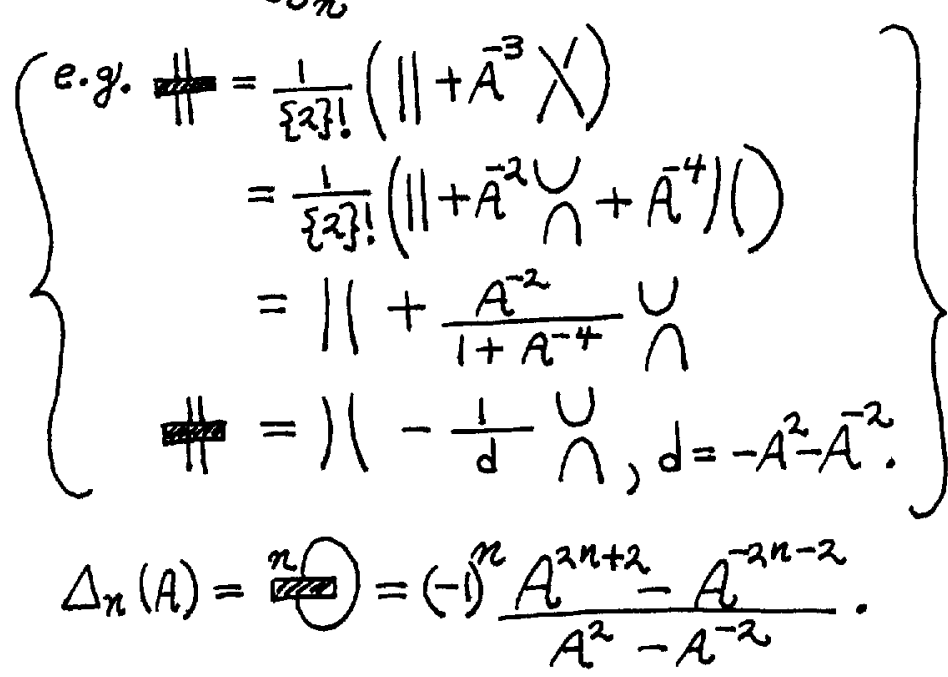

Fig. 9. Deformation

The first combinatorial appearance of these three manifold invariants was in the work of Reshetikhin and Turaev [21] who showed how to construct invariants of three manifolds from invariants of knots and links by using the representation theory of quantum groups. In the case of the quantum group associated with $S U(2)$ (equivalently $S L(2, C)$ ) it is possible to rephrase the Resehtikhin-Turaev work in terms of spin networks associated with the bracket polynomial [12], [13], [14]. It is to this version of the invariant that we refer when we speak of the spin network invariant of three-manifolds.

In Figure 10 we have indicated some of the features of this spin network invariant of three-manifolds. We show the formula for the unnormalised invariant (of the three- 
200

L. H. KAUFFMAN

manifold obtained from surgery on the given link diagram) as a sum over generalised bracket invariants of knots labelled with (q-deformed) antisymmetrizers. A recoupling formula rewrites the crossings in the knot diagrams as sums of trivalent graph evaluations. The result is a formula for the three-manifold invariant in terms of evaluations of trivalent graphs. This is one version of the spin network evaluation for the three-manifold invariant. There are a number of other reformulations of this basic set-up. We refer the reader to [14] for an account of the the Kirillov-Reshetikhin shadow world in this context. This shadow world performs a translation of the knot diagrams into spin nets, showing that the invariant can be computed by a state summation with labels on the regions as well as on the lines. The vertex weights then involve tetrahedral net evaluations at the crossings and theta nets at the edges. This puts the general invariant in line with ( its product with its complex conjugate) the Turaev-Viro invariant. The Turaev-Viro invariant has a beautiful description as a state summation on the triangulation of the three manifold. This gives a direct relationship between the spin network approach and the combinatorial structure
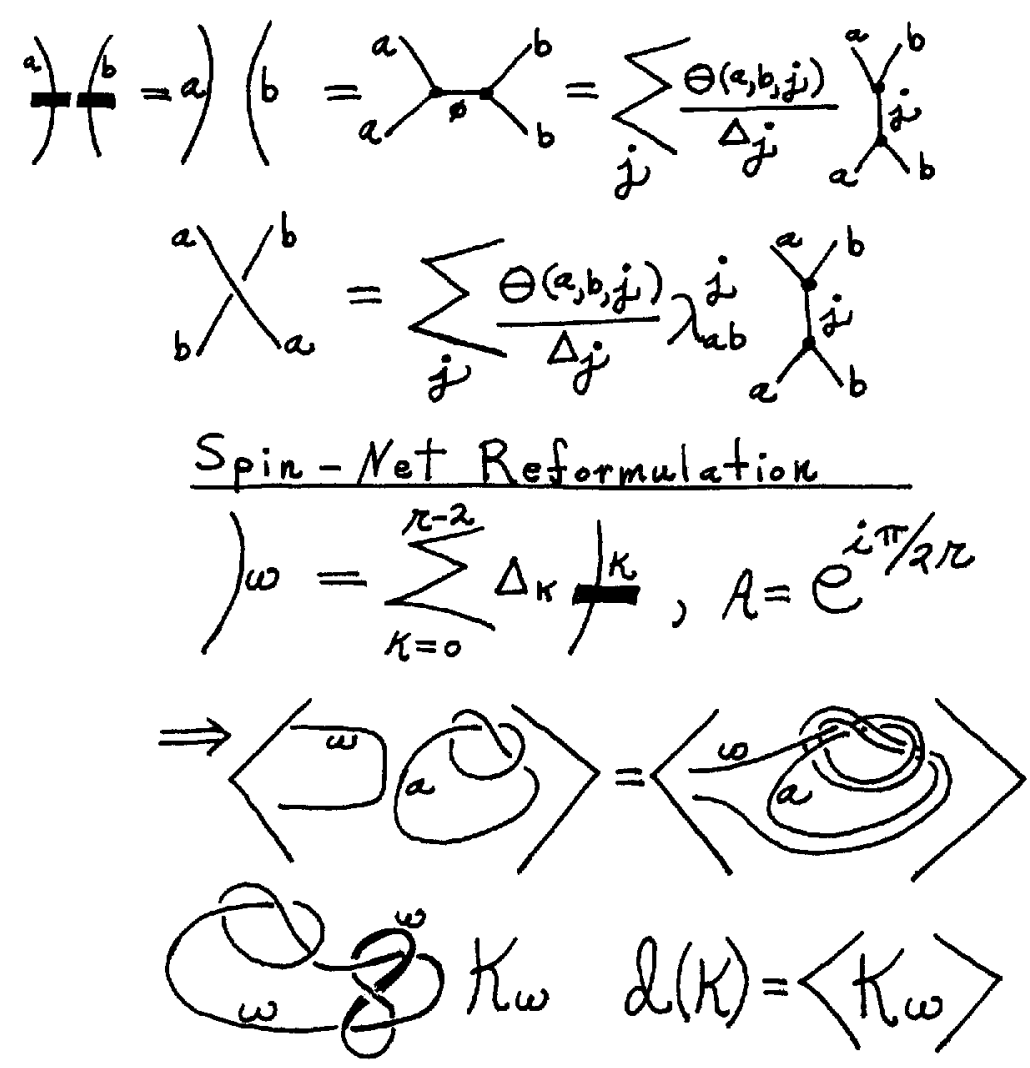

$$
\begin{aligned}
\mathcal{L}(K) & =\begin{array}{l}
\text { unnormalized invariant of } \\
M^{3}(K)
\end{array}=\left\{\begin{array}{l}
3-\text { manifold obtained by } \\
\text { surgery on } K .
\end{array}\right\}
\end{aligned}
$$

Fig. 10. Invariants of three-manifolds 
of the associated three-manifold. It also motivates a generalisation to four-manifolds that gives a state summation formula for the signature of a four manifold (again via the q-deformed spin nets) [5].

If there were a generalisation of the Penrose-Moussouris chromatic evaluation of spin nets to this category, there would be significant global formulas for these invariants. The search for such formulas is tied in with our next topic.

4.1. Witten's functional integral. Simultaneously and independently of Reshetikhin and Turaev, Edward Witten devised a presentation of three-manifold invariants via functional integrals. (Witten had a precursor in the 1978 work of A. Schwarz [23]. Schwarz showed how to express the Ray-Singer-Reidemeister torsion of a three manifold as a functional integral. Witten generalizes Schwarz.) Witten's work adds a significant dimension to the spin network approach. His integral formula is expressed as follows:

$$
Z\left(M^{3}, K\right)=\int d A e^{(i k / 4 \pi){ }_{M}^{\mathrm{T}}} \operatorname{tr}\left(A d A+(2 / 3) A^{3}\right) \operatorname{tr}\left(P e^{\mathrm{D}}{ }^{A}\right) .
$$

The integration is over all gauge potentials modulo gauge equivalence for $S U(2)$ gauge at the fundamental representation. A is a gauge potential on three dimensional space with values in this representation of $S U(2)$. The Chern-Simons Lagrangian,

$$
L=\int_{M} \operatorname{tr}\left(A d A+(2 / 3) A^{3}\right),
$$

provides the formally correct weighting factor against which to integrate the Wilson loop, $\operatorname{tr}\left(P e_{K}^{A}\right)$, to obtain a function $Z\left(M^{3}, K\right)$ of a three manifold M containing a link $\mathrm{K}$. This function is, up to a normalization related to framing, an invariant of the three manifold, link pair. We shall refer to $Z\left(M^{3}, K\right)$ as the Witten invariant of the three-manifold. In particular, we shall write

$$
Z\left(M^{3}\right)=\int d A e^{(i k / 4 \pi)^{\mathrm{T}}}{ }_{M} \operatorname{tr}\left(A d A+(2 / 3) A^{3}\right)
$$

for the corresponding functional, defined on a three manifold without a specified link embedded within it.

The functional integral is, at this point in time, a purely formal approach to this invariant of links and three manifolds. Nevertheless it does follow from Witten's work that the invariant under discussion must be identical to that invariant that we have described as computed from generalised spin networks associated with the bracket polynomial. (If $A=e^{i \pi / 2 r}$, then the coupling constant $k$ in Witten's integral corresponds to $r$ (up to a fixed finite shift).) The verification of this fact is indirect, relying on an understanding of the behaviour of the Witten invariant under surgery on links in the three manifold. It is an open problem to derive this relationship between the Witten invariant and the spin network invariant by an appropriate discretization of the functional integral. Such a derivation would shed light on the nature of both the spin networks and the integration process.

Once the invariant has been expressed in terms of the functional integral, many conjectures and relationships about the spin network evaluations come forth. For example, the large $k$ limit of the functional integral is asymptotically approximated by sums over flat gauge potentials, leading to specific formulas for the asymptotic approximation of the 
spin nets as the order of the root of unity goes to infinity. See [6], [8], [15],[22]. These conjectures have been verified in many special cases, but the general problem remains open. It is quite possible that this problem will be elucidated by an appropriate generalisation of the chromatic method of spin network evaluation [18], [12], [14].

Another remarkable relationship is noted by Witten in [27]. The product of $Z\left(M^{3}\right)$ and its complex conjugate can be expressed as an integral that naturally interprets as a functional integral for $2+1$ quantum gravity with a cosmological constant:

$$
\begin{aligned}
\left|Z\left(M^{3}\right)\right|^{2} & =\int d A d B e^{(i k / 4 \pi)^{\mathrm{T}}} \operatorname{tr}\left(A d A+(2 / 3) A^{3}-B d B-(2 / 3) B^{3}\right) \\
& =\int d e d \omega e^{i^{\mathrm{T}} \operatorname{tr}\left(e R+(\lambda / 3) e^{3}\right)}
\end{aligned}
$$

where $e=(k / 8 \pi)(A-B), \omega=(1 / 2)(A+B), R=d \omega+\omega^{2}$ and $\lambda=(4 \pi / k)^{2}$. Here $e$ is interpreted as a metric. while $\omega$ is interpreted as a connection so that $R$ is the curvature.

We know that this functional integral is a topological invariant (up to standard normalization) of the 3-manifold $M$, and furthermore the invariant can be computed by spin network methods from any triangulation of $M$ via the Turaev-Viro state summation [25] (See also [14]). The first hint that such a state summation might be related to gravity appeared in the work of Regge-Ponzano [19] and Hasslacher-Perry [7]. These authors investigated analogous state sums using approximations to classical recoupling coefficients. They found that the state summations give approximations to simplicial quantum gravity in the Regge calculus. The Chern-Simons functional integral formulation shown above suggests that there should be a simplicial quantum gravity with cosmological constant that corresponds to the topological invariant $\left|Z\left(M^{3}\right)\right|^{2}$. This means that there should be an appropriate formulation of $2+1$ quantum gravity that can be done simplicially and that involves either the deformed spin networks or (equivalently) the quantum group corresponding to $S L(2, C)$ with deformation parameter a root of unity. These are open problems.

$2+1$ quantum gravity is related to a three dimensional topological quantum field theory. The analog for $3+1$ quantum gravity is equally tantalizing, but unfulfilled due to a lack, at present time, of combinatorial models for topological quantum field theories in dimension four. We refer the reader to [4], [3] and [5]. Perhaps amplitudes for 3+1 quantum gravity will correspond to relative topological invariants of four dimensional manifolds.

Finally, we mention the long-standing work of Ashtekar,Rovelli and Smolin that starts with the loop representation of the Ashtekar formulation of quantum gravity. In this theory of quantum gravity the wave functions $\psi$ are functions $\psi(A)$ of (the equivalent of) a gauge potential A on three dimensional space. Smolin and Rovelli consider the loop transform

$$
\hat{\psi}(K)=\int d A \psi(A) \operatorname{tr}\left(P e^{\mathrm{D}}{ }^{A}\right) .
$$

This formal transform rewrites the theory in terms of functionals on knots and links in three dimensional space. Recent work [24] develops quantum gravity directly in terms of knots, links and spin networks. It remains to be seen how this form of quantum gravity will interface with topological quantum field theories in dimension four. 


\section{References}

[1] D. Bullock, Rings of $S L_{2}(C)$ characters and the Kauffman bracket skein module, preprint, 1996.

[2] J. Scott Carter, D. E. Flath and M. Saito, The Classical and Quantum 6j-Symbols, Math. Notes 43, Princeton Univ. Press, 1995.

[3] J. Scott Carter, L. H. Kauffman and M. Saito, Diagrammatics, Singularities and Their Algebraic Interpretations, in: Conference Proceedings of the Brasilian Mathematical Society, to appear.

[4] L. Crane and I. Frenkel, Four dimensional topological quantum field theory, Hopf categories and canonical bases, J. Math. Phys. 35 (1994), 5136-5154.

[5] L. Crane, L. H. Kauffman and D. Yetter, State sum invariants of 4-manifolds, J. Knot Theory and Its Ramifications, 1997, to appear.

[6] D. S. Freed and R. E. Gompf, Computer calculation of Witten's three-manifold invariant, Comm. Math. Phys. 141 (1991), 79-117.

[7] B. Hasslacher and M. J. Perry, Spin networks are simplicial quantum gravity, Phys. Lett. B 103 (1981), 21-24.

[8] L. C. Jeffrey, Chern-Simons-Witten invariants of lens spaces and torus bundles, and the semi-classical approximation, Comm. Math. Phys. 147 (1992), 563-604.

[9] V. F. R. Jones, A polynomial invariant of links via von Neumann algebras, Bull. Amer. Math. Soc. 129 (1985), 103-112.

[10] L. H. Kauffman, State models and the Jones polynomial, Topology 26 (1987), 395-407.

[11] - Statistical mechanics and the Jones polynomial, in: Contemp. Math. 78, Amer. math. Soc., 1989, 263-297.

[12] - Map coloring, q-deformed spin networks, and Turaev-Viro invariants for 3-manifolds, Internat. J. Modern Phys. B 6 (1992), 1765-1794.

[13] —, Knots and Physics, World Scientific, 1991, 1993.

[14] L. H. Kauffman and S. L. Lins, Temperley-Lieb Recoupling Theory and Invariants of 3-Manifolds, Ann. Math. Stud. 114, Princeton Univ. Press, 1994.

[15] R. Lawrence, Asymptotic expansions of Witten-Reshetikhin-Turaev invariants for some simple 3-manifolds, J. Math. Phys. 36 (1995), 6106-6129.

[16] J. P. Moussouris, Quantum Models of Space-Time Based on Recoupling Theory, thesis, Oxford Univ., 1983.

[17] R. Penrose, Angular momentum: An approach to Combinatorial Spacetime, in: Quantum Theory and Beyond, T. Bastin (ed.), Cambridge Univ. Press, 1969.

[18] —, Applications of negative dimensional tensors, in: Combinatorial Mathematics and Its Applications, D. J. A. Welsh (ed.), Academic Press, 1971.

[19] G. Ponzano and T. Regge, Semiclassical limit of Racah coefficients, in: Spectroscopic and Group Theoretical Methods in Theoretical Physics, North-Holland, Amsterdam, 1968.

[20] K. Reidemeister, Knotentheorie, Julius Springer, Berlin, 1933; Chelsea, N.Y., 1948.

[21] N. Y. Reshetikhin and V. Turaev, Invariants of Three Manifolds via link polynomials and quantum groups, Invent. Math. 103 (1991), 547-597.

[22] L. Rozansky, Witten's invariant of 3-dimensional manifolds: loop expansion and surgery calculus, in: Knots and Applications, L. Kauffman (ed.), World Scientific, 1995.

[23] A. Schwarz, The partition function of degenerate quadratic functional and Ray-Singer invariants, Lett. Math. Phys. 2 (1978), 247. 
[24] L. Smolin, The geometry of quantum spin networks, preprint, Center for Gravitational Physics and Geometry, Penn. State University, University Park, PA, 1996.

[25] V. G. Turaev and O. Y. Viro, State sum invariants of 3-manifolds and quantum $6 j$ symbols, Topology 31 (1992), 865-902.

[26] E. Witten, Quantum field theory and the Jones polynomial, Comm. Math. Phys. 121 (1989), 351-399.

[27] - 2+1 gravity as an exactly soluble system, Nuclear Phys. B 311, (1988/89), 46-78. 